\title{
FLUID FLOW AND INERTIAL FORCES IN BLACK HOLE SPACE TIMES
}

\author{
BANIBRATA MUKHOPADHYAY \& A. R. PRASANNA \\ Theoretical Physics Group, Physical Research Laboratory, Navrangpura, \\ Ahmedabad-380009, India
}

\begin{abstract}
We discuss the nature of the radial and azimuthal components of centrifugal force associated with fluid flows in the equatorial plane of black hole space times. The equations of motion are solved for the radial and azimuthal components of the 3velocity $V^{i}$ which are then used in evaluating the nature of the various components of inertial accelerations. It is shown that the reversal of centrifugal force is governed mainly by the dominance of the azimuthal velocity and the reversal occurs for $r$, mostly at $2 m \lesssim r \lesssim 3 m$, depending upon the boundary condition.
\end{abstract}

\section{International Journal of Modern Physics A}

\section{Introduction}

Most of the discussion on fluid flows in astrophysical context are Newtonian 1 . 3 . 3 in nature, with a few considering post Newtonian features 4.6 . However, if the flows are to be considered in the context of black holes, it is only natural to expect the effects of space time curvature to affect the flows considered in a general relativistic framework. Such considerations may indeed show new features, quite unfamiliar in pure Newtonian descriptions. One of the ways to study such features is to develop a full scale general relativistic formalism of flows around black holes. However, there is one aspect of general relativity which yields the possibility of looking for Newtonian features, through a partitioning of space time in a $3+1$ language. It has been shown by Abramowicz et al. 8 that one can indeed introduce the concept of 'inertial forces' in general relativity through a covariant formalism, as applied to test particle motion in curved space time. Recently Prasanna 1 (hereafter refers to as Paper I), using a matching of the $3+1$ conformal splitting of space-time (optical reference geometry 10 ), with the ADM splitting 11 in terms of the lapse function $\alpha$ and shift vector $\beta^{i}$ has expressed the components of the covariantly defined centrifugal and Coriolis forces acting on a fluid element in the given curved space time to be

$$
(C f)_{i}=\gamma^{2}\left[V V^{j} \partial_{j}\left(\frac{V_{i}}{V}\right)+\left(V_{i} V^{j} \partial_{j}-V^{2} \partial_{i}\right) \Phi-\frac{1}{2} V^{j} V^{k} \partial_{i} \gamma_{j k}\right]
$$

and

$$
(C o)_{i}=-\frac{\gamma^{2}}{\alpha}\left[V \beta^{j} \partial_{j}\left(\frac{V_{i}}{V}\right)+V^{j} \partial_{i} g_{0 j}-\beta^{k} V^{j} \partial_{i} \gamma_{k j}\right]
$$

where,

$$
V^{2}=\gamma_{i j} V^{i} V^{j} \quad \text { and } \quad \gamma=\frac{1}{\sqrt{1-V^{2}}}
$$




$$
V^{i}=\frac{\left(\frac{u^{i}}{u^{t}}+\beta^{i}\right)}{\alpha} .
$$

Here, $u^{\mu}=\left(u^{t}, u^{i}\right)$ is the four velocity of the fluid element and $\Phi$ is the gravitational potential of the back ground space time. Further it was shown in Paper I 9 that in static space time for the case of pure dust fluid (pressure zero) the centrifugal force would show reversal only when $V^{\phi}$ is non zero whereas when both $V^{r}$ and $V^{\phi}$ are non zero there is no reversal. It is known that generally in most of the stationary flows the radial component of the velocity vector is non zero and one should see what happens to the force components if the pressure is non zero and consequently the gradient of pressure also comes into action. It is possible that the gradient of pressure may balance the force due to radial velocity and thereby allow the azimuthal component of the velocity to dominate and consequently affect the centrifugal force.

With this in mind, we now consider the study of inertial forces for general flows with pressure non zero and with different equations of state. We shall in this consider the flows essentially confined to the equatorial plane $\theta=\pi / 2$ and evaluate the inertial force components, after solving for the velocity fields from the standard equation of motion on a given background geometry.

\section{Formalism}

The general equations of motion for a perfect fluid on curved space time are given by:

$$
(\rho+P) u_{; \nu}^{\mu} u^{\nu}+h^{\mu \nu} P_{\nu}=0
$$

Using the definition of 3-velocity $V^{i}$, one can write explicitly the equation of continuity

$$
\begin{gathered}
(\rho+P)\left(u^{t}\right)^{2}\left[\frac{\partial}{\partial x^{i}}\left(\alpha V^{i}-\beta^{i}\right)+\Gamma_{o \nu}^{\nu}+\Gamma_{j \nu}^{\nu}\left(\alpha V^{j}-\beta^{i}\right)-\Gamma_{o o}^{o}\right. \\
\left.-2 \Gamma_{o j}^{o}\left(\alpha V^{j}-\beta^{j}\right)-\Gamma_{j k}^{o}\left(\alpha V^{j}-\beta^{j}\right)\left(\alpha V^{k}-\beta^{k}\right)\right] \\
+\left(u^{t}\right)^{2}\left[\frac{\partial}{\partial t}(\rho-P)+\left(\alpha V^{j}-\beta^{j}\right) \frac{\partial}{\partial x^{j}}(\rho-P)\right]=g^{o \nu} \frac{\partial P}{\partial x^{\nu}}
\end{gathered}
$$

and the momentum equations

$$
\begin{gathered}
(\rho+P)\left(u^{t}\right)^{2}\left[\frac{\partial}{\partial t}\left(\alpha V^{i}-\beta^{i}\right)+\left(\alpha V^{j}-\beta^{j}\right) \frac{\partial}{\partial x^{j}}\left(\alpha V^{i}-\beta^{i}\right)\right. \\
+\Gamma_{o o}^{i}-\Gamma_{o o}^{o}\left(\alpha V^{i}-\beta^{i}\right)+2\left(\alpha V^{j}-\beta^{j}\right)\left(\Gamma_{o j}^{i}-\Gamma_{o j}^{o}\left(\alpha V^{i}-\beta^{i}\right)\right) \\
\left.\left(\alpha V^{j}-\beta^{j}\right)\left(\alpha V^{k}-\beta^{k}\right)\left(\Gamma_{j k}^{i}-\Gamma_{j k}^{o}\left(\alpha V^{i}-\beta^{i}\right)\right)\right]
\end{gathered}
$$




$$
=\left(\left(\alpha V^{i}-\beta^{i}\right) g^{o \nu}-g^{i \nu}\right) \frac{\partial P}{\partial x^{\nu}} .
$$

We shall presently consider these on a stationary back ground as specified by the Kerr geometry in Boyer-Lindquist frame:

$$
d s^{2}=-\frac{\left(\Delta-a^{2} \sin ^{2} \theta\right)}{\rho^{2}} d t^{2}-4 a \frac{m r \sin ^{2} \theta}{\rho^{2}} d t d \phi+\frac{\rho^{2}}{\Delta} d r^{2}+\rho^{2} d \theta^{2}+\frac{\Sigma}{\rho^{2}} \sin ^{2} \theta d \phi^{2}
$$

with $\Delta=r^{2}-2 m r+a^{2}, \rho^{2}=r^{2}+a^{2} \cos ^{2} \theta, \Sigma=\left(r^{2}+a^{2}\right)^{2}-a^{2} \Delta \sin ^{2} \theta$.

The various components of the shift vectors $\beta_{i}$ and the 3 -metric $\gamma_{i j}$, alongwith the lapse function $\alpha$ are given by (on the plane $\theta=\pi / 2$ ),

$$
\begin{gathered}
\alpha^{2}=\frac{r^{2}+a^{2}-2 m r}{r^{2}+a^{2}+2 m a^{2} / r}, \\
\beta_{i}=(0,0,0,-2 a m / r), \\
\gamma_{r r}=\left(1-\frac{2 m}{r}+\frac{a^{2}}{r^{2}}\right)^{-1} \text { and } \gamma_{\phi \phi}=r^{2}+a^{2}+\frac{2 m a^{2}}{r}
\end{gathered}
$$

while the gravitational potential $\Phi$ is given as

$$
\Phi=-\frac{1}{2} \ln \left[\frac{r^{3}+a^{2} r-2 m r^{2}}{r^{3}+a^{2} r+2 m a^{2}}\right] .
$$

Using these in the general equations referred to, alongwith the components of connection coefficients for the metric (9) one gets, the momentum equation:

$$
\begin{gathered}
(P+\rho)\left[\bar{u}^{r} \frac{d \bar{u}^{r}}{d r}+\left\{\frac{a^{2}-m r}{\Delta r}-\frac{2 m\left(a^{2}+r^{2}\right)}{\Delta r^{2}}\right\}\left(\bar{u}^{r}\right)^{2}+\frac{2 a m}{\Delta r^{2}}\left(a^{2}+3 r^{2}\right)\left(\bar{u}^{r}\right)^{2} \bar{u}^{\phi}(1\right. \\
\left.+\frac{\Delta}{r^{4}}\left(m a^{2}-r^{3}\right)\left(\bar{u}^{\phi}\right)^{2}-\frac{2 m a \Delta}{r^{4}} \bar{u}^{\phi}+\frac{\Delta m}{r^{4}}\right] \\
=\frac{\Delta}{r^{2}}\left[-\frac{\Delta-a^{2}}{r^{2}}+\frac{r^{2}}{\Delta}\left(\bar{u}^{r}\right)^{2}-\frac{4 a m}{r} \bar{u}^{\phi}+\frac{\left(r^{2}+a^{2}\right)^{2}-a^{2} \Delta}{r^{2}}\left(\bar{u}^{\phi}\right)^{2}\right] \frac{d P}{d r} \\
\frac{d \bar{u}^{\phi}}{d r}+\frac{2 a m}{\Delta r^{2}}\left(a^{2}+3 r^{2}\right)\left(\bar{u}^{\phi}\right)^{2}-\frac{2}{\Delta r^{2}}\left(2 m a^{2}+r^{2}(3 m-r)\right) \bar{u}^{\phi}+\frac{2 m a}{\Delta r^{2}}=0
\end{gathered}
$$

and the equation of continuity:

$$
\begin{array}{r}
\frac{1}{\bar{u}^{r}} \frac{d \bar{u}^{r}}{d r}+\frac{2 a m}{\Delta r^{2}}\left(a^{2}+3 r^{2}\right) \bar{u}^{\phi}-\frac{m}{\Delta r^{2}}\left(a^{2}+r^{2}\right)+\frac{1}{r \Delta}\left(a^{2}-m r\right) \\
-\frac{1}{\Delta r^{2}}\left(m a^{2}+r^{2}(2 m-r)\right)=\frac{1}{(P+\rho)} \frac{d}{d r}(P-\rho),
\end{array}
$$

where, 


$$
\bar{u}^{i}=\frac{u^{i}}{u^{t}}=\alpha V^{i}-\beta^{i} .
$$

We shall consider the flows with different equation of state and look at the behaviour of the force components. For the present we are restricting ourselves to the case of flows on the equatorial plane $(\theta=\pi / 2)$ of the central gravitating source, a condition which has been incorporated in the equations above.

\section{Solutions}

Case (1), P=0; Dusty Fluid

As this refers to the case when streamlines are geodesics, the results are same as obtained in the case of test particles, considered in 'Paper I'.

Case (2), $\rho=$ constant

\section{(a) Schwarzschild space time}

We shall first consider the static case by putting $a=0$ and consequently have the equations:

$$
\begin{gathered}
\alpha V^{r} \frac{d}{d r}\left(\alpha V^{r}\right)+\frac{m}{r^{2}}\left(1-\frac{2 m}{r}\right)-\frac{3 m}{r^{2}}\left(1-\frac{2 m}{r}\right)^{-1}\left(\alpha V^{r}\right)^{2}-(r-2 m)\left(\alpha V^{\phi}\right)^{2}(18) \\
=-\frac{1}{\left(u^{t}\right)^{2}}\left(1-\frac{2 m}{r}\right) \frac{1}{(P+\rho)} \frac{d P}{d r} \\
\frac{d}{d r}\left(\alpha V^{\phi}\right)+\frac{2}{r}\left(1-\frac{2 m}{r}\right)^{-1}\left(1-\frac{3 m}{r}\right) \alpha V^{\phi}=0
\end{gathered}
$$

and the equation of continuity:

$$
\frac{d}{d r}\left(\alpha V^{r}\right)+\frac{2}{r}\left(1-\frac{2 m}{r}\right)^{-1}\left(1-\frac{3 m}{r}\right) \alpha V^{r}=\frac{\alpha V^{r}}{(P+\rho)} \frac{d P}{d r} .
$$

Thus one finds $\alpha V^{\phi}=\frac{l}{r^{2}}\left(1-\frac{2 m}{r}\right)$ and $\alpha V^{r}$ satisfies the equation

$$
\begin{array}{r}
-\left(1-\frac{2 m}{r}\right)^{2}\left[1-\frac{l^{2}}{r^{2}}\left(1-\frac{2 m}{r}\right)\right] \frac{1}{\alpha V^{r}} \frac{d}{d r}\left(\alpha V^{r}\right)+\frac{2}{r}\left(1-\frac{3 m}{2 r}\right)\left(1-\frac{2 m}{r}\right)^{-1}\left(\alpha V^{r}\left(2^{2} 1\right)\right. \\
+\left(1-\frac{2 m}{r}\right)\left[-\frac{2}{r}+\frac{5 m}{r^{2}}+\frac{l^{2}}{r^{3}}\left(1-\frac{2 m}{r}\right)\left(3-\frac{8 m}{r}\right)\right]=0 .
\end{array}
$$

Solving which one can get

$$
\left(\alpha V^{r}\right)^{2}=\frac{\left(1-\frac{2 m}{r}\right)^{2}\left[1-\frac{l^{2}}{r^{2}}\left(1-\frac{2 m}{r}\right)\right]}{\left[1+c_{1} r^{4}\left(1-\frac{2 m}{r}\right)\right]},
$$


where $c_{1}$ is the constant of integration to be chosen appropriately. The components of centrifugal force are then given by

$$
\begin{aligned}
(C f)_{r} & =\frac{l^{2}\left[(2 m-r)\left(c_{1} r^{2}\left(6 m^{2}-5 r m+r^{2}\right)-1\right) l^{2}+(3 m-2 r) r^{2}\right]}{r^{3}\left(r^{3}+l^{2}(2 m-r)\right)\left(c_{1} l^{2}(r-2 m)^{2}+1\right)} \\
(C f)_{\phi} & =-\frac{l\left[(2 m-r)\left(c_{1} r^{2}\left(6 m^{2}-5 r m+r^{2}\right)-1\right) l^{2}+(3 m-2 r) r^{2}\right]}{r^{4} \sqrt{\frac{r^{3}+l^{2}(2 m-r)}{r^{3}}} \sqrt{c_{1}(r-2 m) r^{3}+1}\left(c_{1} l^{2}(r-2 m)^{2}+1\right)} .
\end{aligned}
$$

It should be noted at the outset that for $l=3 \sqrt{3} m$, at $r=3 m$, the total velocity $V$ tends to 1 , the velocity of light and thus makes the Lorentz factor infinite. This feature is independent of the constant $c_{1}$ as may be seen from the expression for $V$ $\left(=\left[1+c_{1} l^{2}(r-2 m)^{2}\right] /\left[1+c_{1} r^{3}(r-2 m)\right]\right)$. Thus, restricting the value of $l<3 \sqrt{3} m$,

it may be seen that the centrifugal reversal occurs for two values of $r$ one close to $r=3 m$ and other close to the horizon $r=2 m$, depending upon the choice of $l$ and $c_{1}$. For very low values of $l$ and $c_{1}$ the real roots may all lie within the horizon (e.g. for $l=1$ and $\left.c_{1}=6, r_{1}=1.73, r_{2}=0.175, r_{3}=-0.153\right)$. In principle there are five zeros for the quintic equation obtainable by setting $C f=0$, but only two of them lie outside the horizon. For a fixed $l$ as $c_{1}$ is increased, the outer most root stays fixed at $r=3 m$, while the other one moves closer and closer to the horizon. Figures 1 gives the plots for the components of the centrifugal force for different values of $l$ and $c_{1}$.

\section{(b) Kerr space time}

The equations of motion for this background with $\rho=$ constant may be obtained from the general equations (14), (15) and (16). Though the equation for the azimuthal velocity is decoupled from the other two, the equations are not tractable to solve analytically. Thus integrating them numerically with the appropriate boundary conditions one can obtain the profiles for the velocity, using which the profiles for the force components may be obtained. Figure 2 gives the plots for the various profiles for different values of the Kerr parameter $a$. As may be seen, there is a definite reversal of both components of the centrifugal force in each case at locations close to $r \leq 3 m$ (see Table 1). The pressure profile shows a monotonic increase close to the central compact object.

Case (3), $\rho=P$; Isothermal Fluid

\section{(a) Schwarzschild space time}

In the extreme case of isothermal equation of state the equations of motion on the Schwarzschild background reduce to

$$
\begin{aligned}
2 P\left(u^{t}\right)^{2}\left[\alpha V^{r} \frac{d}{d r}\left(\alpha V^{r}\right)+\frac{m}{r^{2}}\right. & \left(1-\frac{2 m}{r}\right)-\frac{3 m}{r^{2}}\left(1-\frac{2 m}{r}\right)^{-1}\left(\alpha V^{r}\right)^{2} \\
\left.-(r-2 m)\left(\alpha V^{\phi}\right)^{2}\right] & =-\left(1-\frac{2 m}{r}\right) \frac{d P}{d r}
\end{aligned}
$$

prepared by Latex2e October 27, 2018 

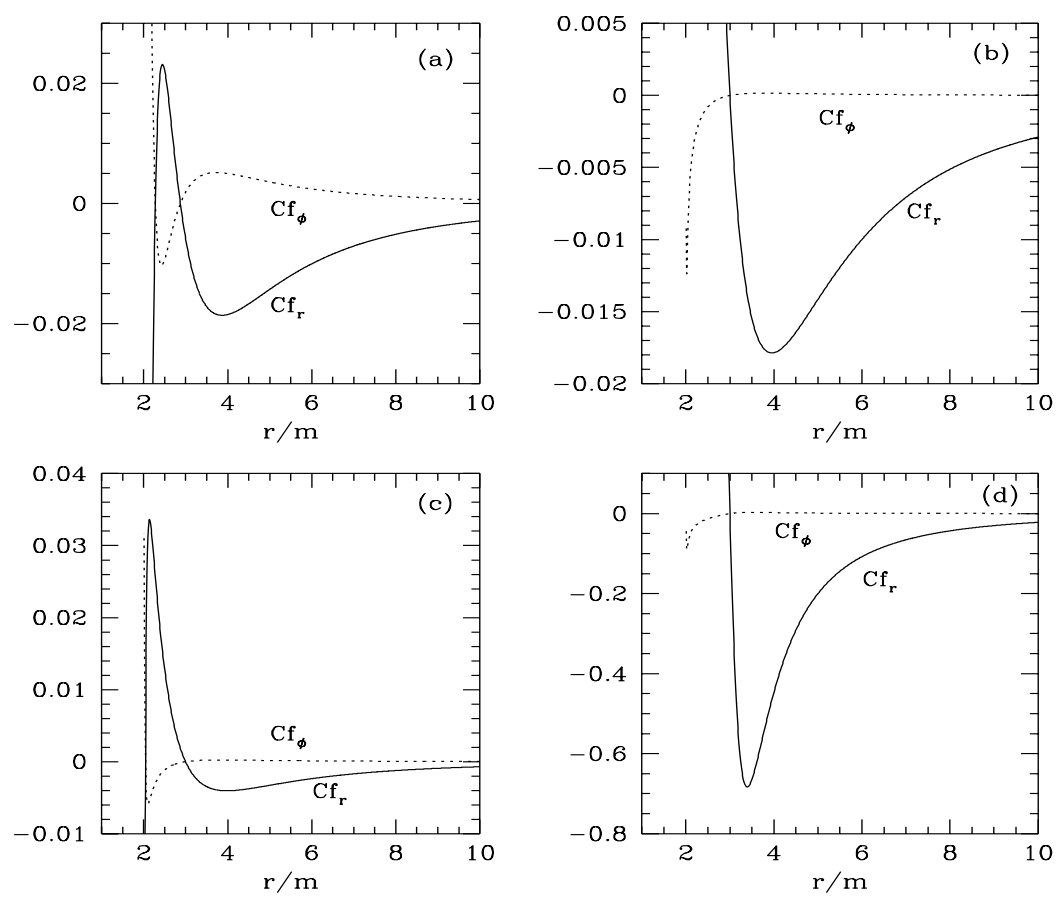

Fig. $1: \rho=$ constant, Schwarzschild case. It shows the variation of $C f_{r}$ and $C f_{\phi}$ as functions of $r / m$ for (a) $l=2$ and $c_{1}=6$, (b) $l=2$ and $c_{1}=6000$, (c) $l=1$ and $c_{1}=600$, (d) $l=5$ and $c_{1}=600$.

$$
\begin{aligned}
& \frac{d}{d r}\left(\alpha V^{\phi}\right)+\frac{2}{r}\left(1-\frac{2 m}{r}\right)^{-1}\left(1-\frac{3 m}{r}\right) \alpha V^{\phi}=0 \\
& \frac{d}{d r}\left(\alpha V^{r}\right)+\frac{2}{r}\left(1-\frac{2 m}{r}\right)^{-1}\left(1-\frac{3 m}{r}\right) \alpha V^{r}=0
\end{aligned}
$$

and

$$
\left(u^{t}\right)^{-2}=\left[\left(1-\frac{2 m}{r}\right)-\left(1-\frac{2 m}{r}\right)^{-1}\left(\alpha V^{r}\right)^{2}-r^{2}\left(\alpha V^{\phi}\right)^{2}\right] .
$$

It is seen from (26) that the angular velocity shows the same behaviour as in Case 2 (a) with $\alpha V^{\phi}=\frac{l}{r^{2}}\left(1-\frac{2 m}{r}\right)$. The radial velocity comes out constrained from the continuity equation equation to be $\alpha V^{r}=\frac{v_{1}}{r^{2}}\left(1-\frac{2 m}{r}\right), v_{1}$ being constant of integration, which by dimensional considerations and also due to the consistency with the equation of continuity can be identified with the ratio $\dot{M} / \rho, \dot{M}$ being the accretion rate and $\rho$ the density. With these, the components of centrifugal force turn out to be

prepared by Latex2e October 27, 2018 

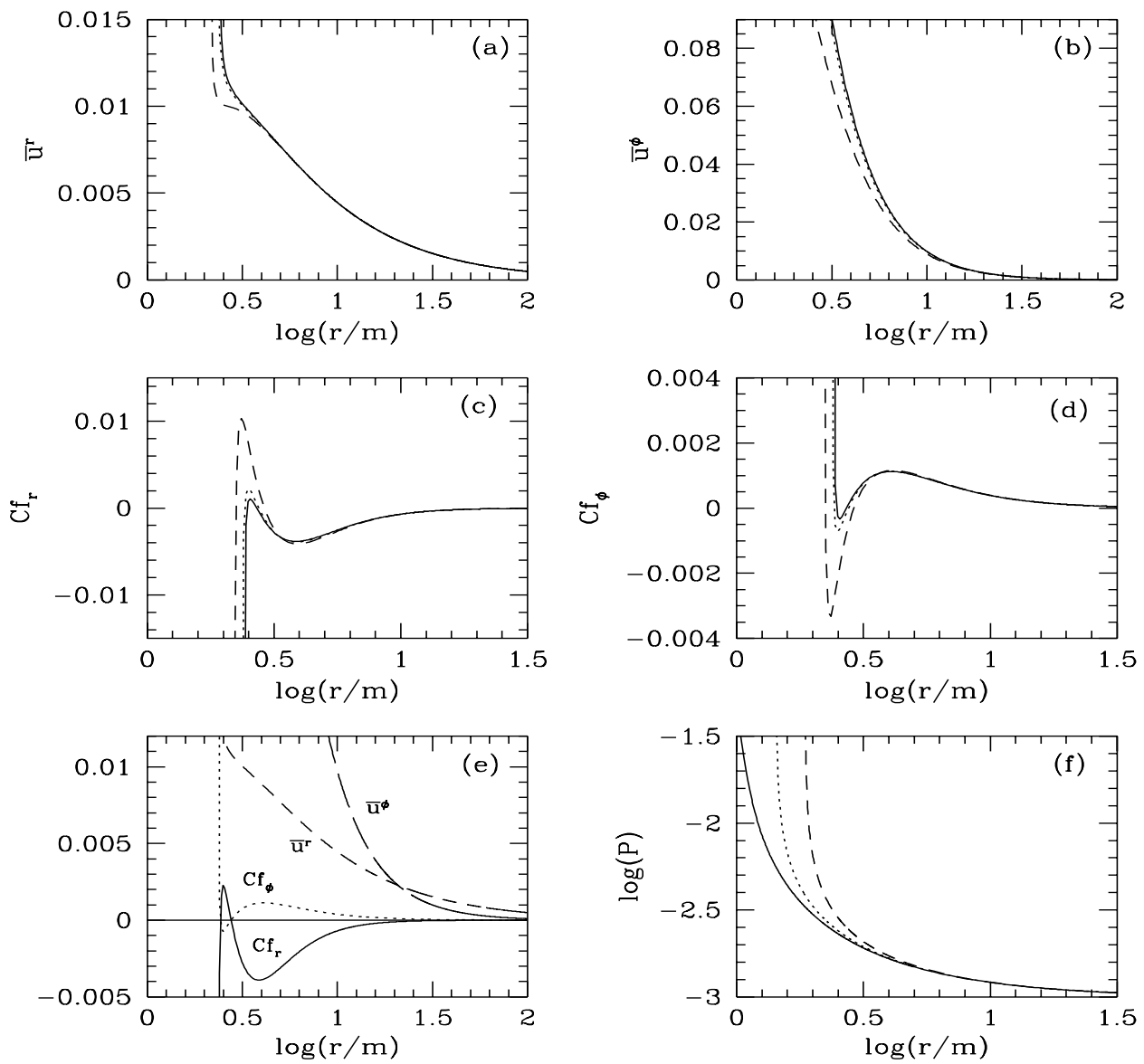

Fig. 2: $\rho=$ constant, Kerr case. It shows the variation of (a) $\bar{u}^{r}$, (b) $\bar{u}^{\phi}$, (c) $C f_{r}$, (d) $C f_{\phi}$ as functions of radial distance in unit of mass of the black hole $(r / m)$. Solid, dotted and dashed curves indicate the results for Kerr parameter $a=1,0.9$ and 0.5 respectively. (e) Variation of $\bar{u}^{r}, \bar{u}^{\phi}, C f_{r}$ and $C f_{\phi}$ as a function of $r / m$ for $a=0.9$ which shows the relation of the centrifugal reversal location with the location where $\bar{u}^{\phi}$ crosses over $\bar{u}^{r}$. (f) Variation of pressure $P$ as a function of radial distance $r / m$.

$$
\begin{aligned}
& (C f)_{r}=-\frac{\gamma^{2} l^{2}}{V^{2} r^{5}}\left(1-\frac{2 m}{r}\right)\left[\frac{2 v_{1}^{2}}{r^{2}}+l^{2}\left(1-\frac{3 m}{r}\right)\right], \\
& (C f)_{\phi}=\frac{\gamma^{2} l v_{1}}{V^{2} r^{3}}\left(1-\frac{2 m}{r}\right)\left[\frac{2 v_{1}^{2}}{r^{2}}+l^{2}\left(1-\frac{3 m}{r}\right)\right]
\end{aligned}
$$

with $\gamma^{2}=\left(1-V^{2}\right)^{-1}$ and $V^{2}=V^{r} V_{r}+V^{\phi} V_{\phi}$. From these expressions it is again clear that these do not change sign for any $r>3 m$. However, for $r<3 m$, both components of the centrifugal force change sign for $r=\frac{3 m}{2}\left(1 \pm \sqrt{1-\frac{8 v_{1}^{2}}{9 l^{2} m^{2}}}\right)$. 

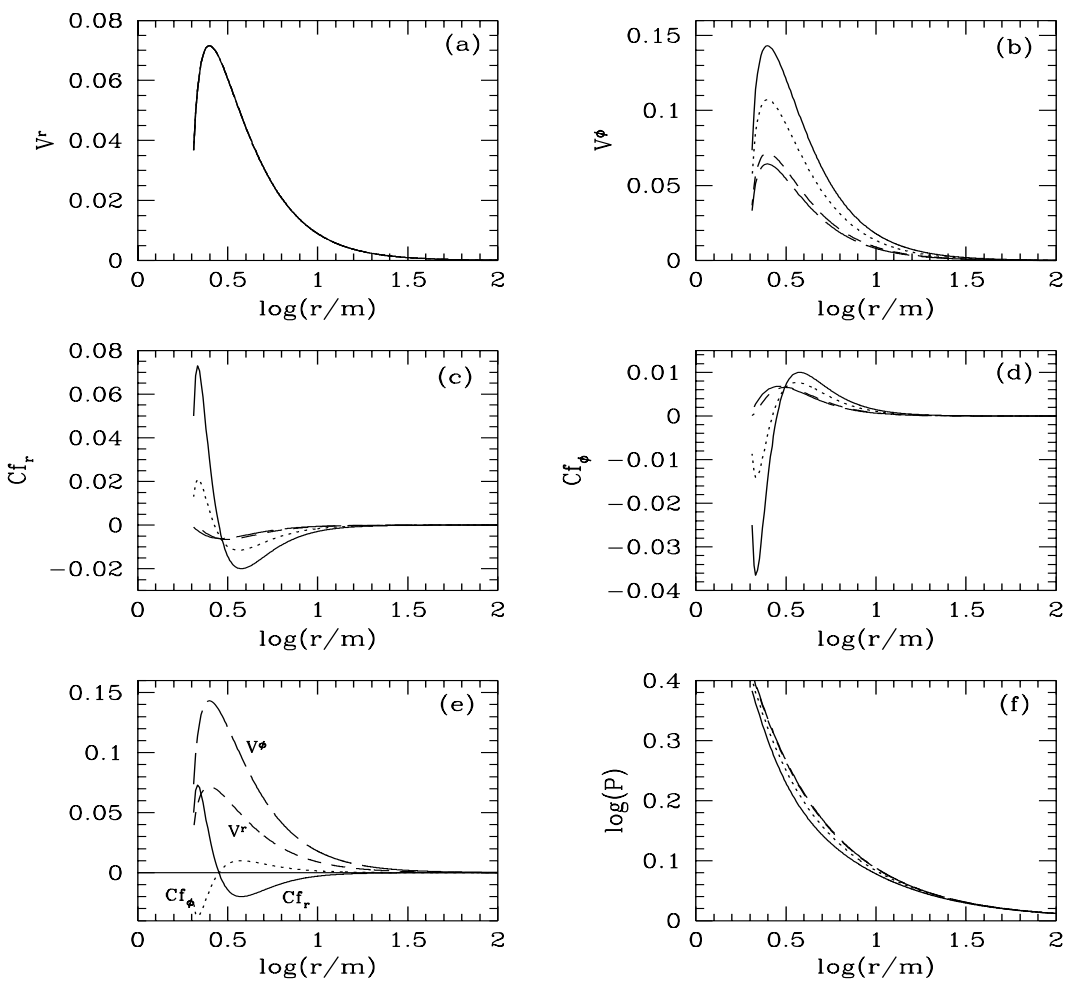

Fig. 3: $P=\rho$, Schwarzschild case. It shows the variation of (a) $V^{r}$, (b) $V^{\phi}$, (c) $C f_{r}$, (d) $C f_{\phi}$ as functions of radial distance in unit of mass of the black hole $(r / m)$. Solid, dotted, dashed and long-dashed curves indicate the results for $l / v_{1}=2,1.5,1$ and 0.9 respectively. (e) Variation of $V^{r}, V^{\phi}, C f_{r}$ and $C f_{\phi}$ as functions of $r / m$ for $l / v_{1}=2$, which shows the dominance of azimuthal velocity over radial velocity and the reversal of centrifugal forces. (f) Variation of pressure $P$ as a function of $r / m$.

Obviously the reversal depends upon the reality of $r$, which demands $\frac{\dot{M}}{\rho} \leq \frac{3 l m}{2 \sqrt{2}}$. Figure 3 shows the profiles of velocity and centrifugal forces for four different values of the ratio $\frac{l m}{v_{1}}$. For $\frac{l m}{v_{1}} \leq 1$ the root lies inside the horizon $r=2 m$ and then the reversal can not be seen. On the other hand for $\frac{l m}{v_{1}}>1$ the root lies outside the horizon $r=2 m$ but at the location $<3 m$.

\section{(b) Kerr space time}

In the case of Kerr background, again the equations are integrated numerically and the profiles presented in Fig. 4. The plots give again the velocity and force components alongwith the pressure profile for different values of $a$. Here again as in the case of $\rho$ constant, the centrifugal force changes sign at a radius $r$ between $2 m$ and $3 m$, the exact location of the zero depending upon the boundary condition (see Table 2). 

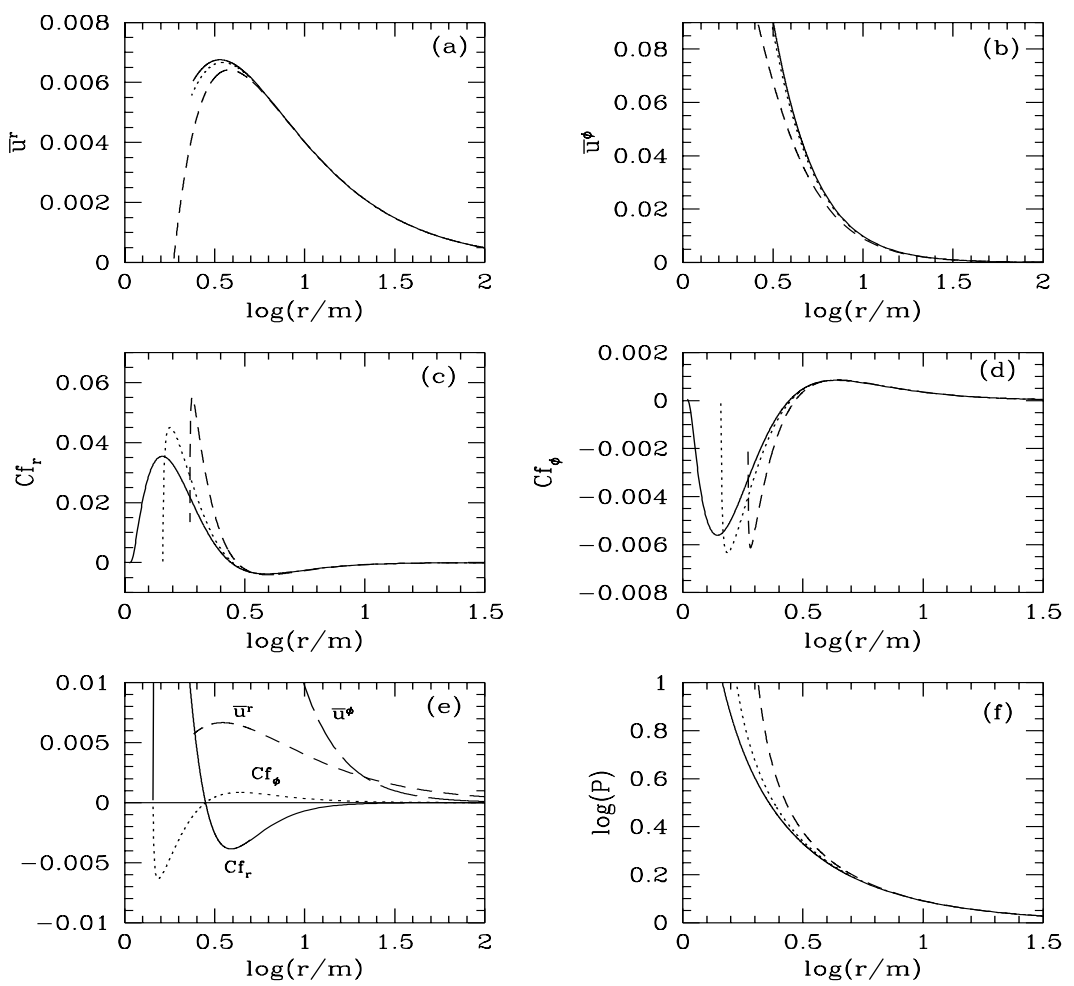

Fig. 4: $P=\rho$, Kerr case. Plots are similar to the ones as Fig. 3.

Case (4), $P=\rho^{\Gamma} ; \Gamma$ being the gas constant

The adiabatic equation of state yields for the static space time, the fluid equations which are coupled for $V^{r}$ and $P$, while the azimuthal component of the velocity again has the same profile $V^{\phi}=\frac{l}{r^{2}}\left(1-\frac{2 m}{r}\right)^{1 / 2}$. The equations for $V^{r}$ and $P$ are solved numerically for different values of $\Gamma$ and the results are presented in Fig. 5 for three different values of $\Gamma ; 5 / 3,4 / 3$ and 1.0001 . The radial velocity profile shows different slopes for different values of the gas constant

which behaviour is reflected in the profiles for pressure and density too. The azimuthal velocity profile is independent of $\Gamma$. Again the centrifugal force shows reversal in the region $2 m<r<3 m$, in accordance with other cases. Though both components of the velocity show a decreasing trend, calculations show that the total velocity $V$ tends toward $c(=1)$ as $r \rightarrow 2 m$, which is in accordance with expectations.

Figures 6 and 7 show the profiles for the same physical quantities in the case of Kerr background for the two distinct cases, $\Gamma=4 / 3$ and $\Gamma=5 / 3$ and for different values of the Kerr parameters ' $a$ '. The behaviour of various quantities are just similar, again showing the feature of centrifugal reversal in the region $2 m<r \leq 3 m$ which is outside the ergo region.

prepared by Latex2e October 27, 2018 

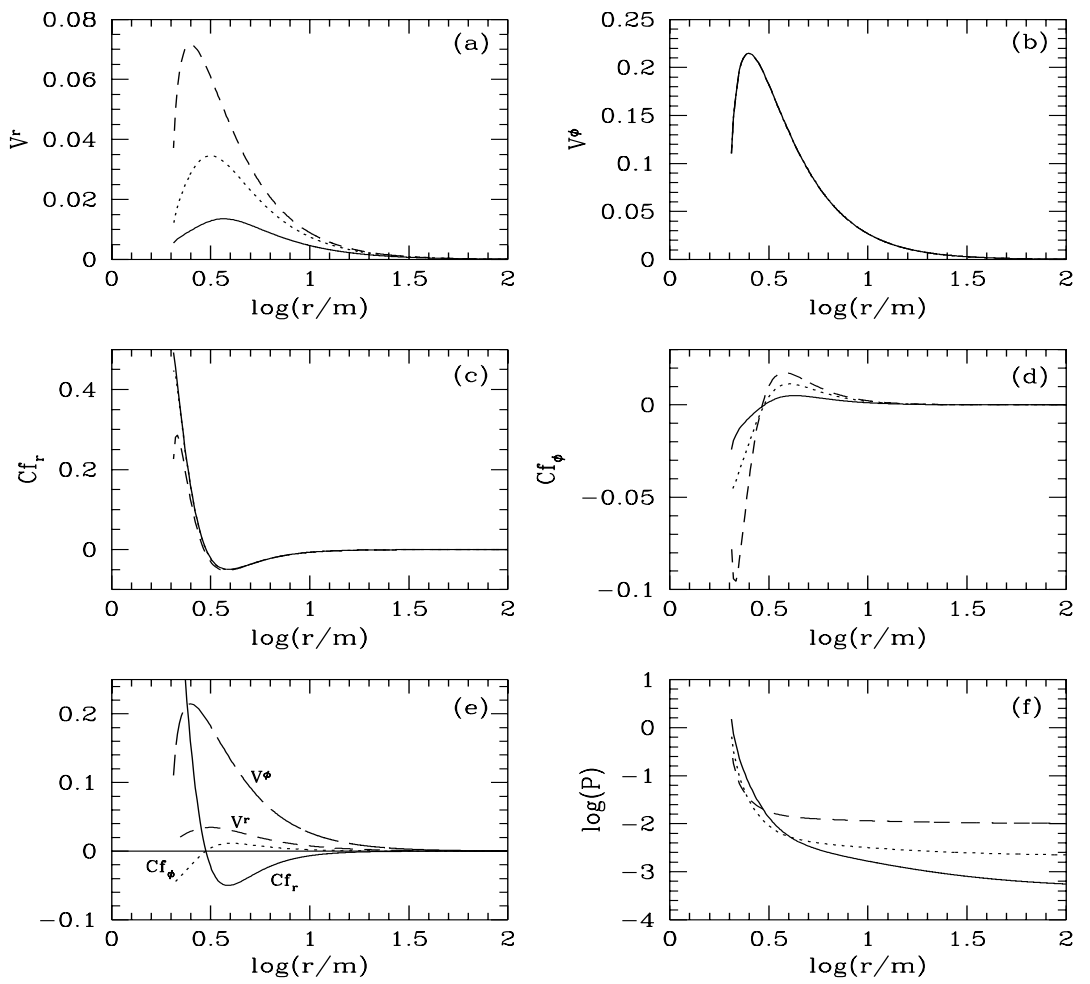

Fig. 5 : Schwarzschild case for adiabatic equation of state. It shows the variation of (a) $V^{r}$, (b) $V^{\phi}$, (c) $C f_{r}$, (d) $C f_{\phi}$, (f) $P$ as functions of radial distance in unit of mass of the black hole $(r / m)$. Solid, dotted and dashed curves indicate the results for gas constant $\Gamma=5 / 3,4 / 3,1.0001$ respectively. (e) Variation of $V^{r}, V^{\phi}, C f_{r}$ and $C f_{\phi}$ as functions of $r / m$ for $\Gamma=4 / 3$ which indicates the dominance of azimuthal velocity over radial velocity and the reversal of centrifugal forces.

\section{Discussion}

We shall first consider the azimuthal component of the centrifugal force in the Schwarzschild geometry for the case of dusty fluid, which turns out to be

$$
(C f)_{\phi}=\frac{l}{2 r}\left[\frac{2 m c^{2}}{r}-\frac{l^{2}}{r^{2}}\left(1-\frac{2 m}{r}\right)\right]^{1 / 2}
$$

and shows no reversal just as the radial component as considered earlier in Paper I 6. On the other hand when we consider the Kerr background the force components do show a reversal even when $P=0$. Obviously the extra contribution towards angular velocity comes from the dragging of inertial frames, an effect that dominates as $r \rightarrow 2 m$ the ergo surface. Thus one finds the reversal of the centrifugal force to occur close to the location where $\bar{u}^{\phi}$ crosses $\bar{u}^{r}$.

When the density is constant with pressure non zero, the analytical expressions are obtained for the components of the centrifugal force in the static case, which

prepared by Latex2e October 27, 2018 

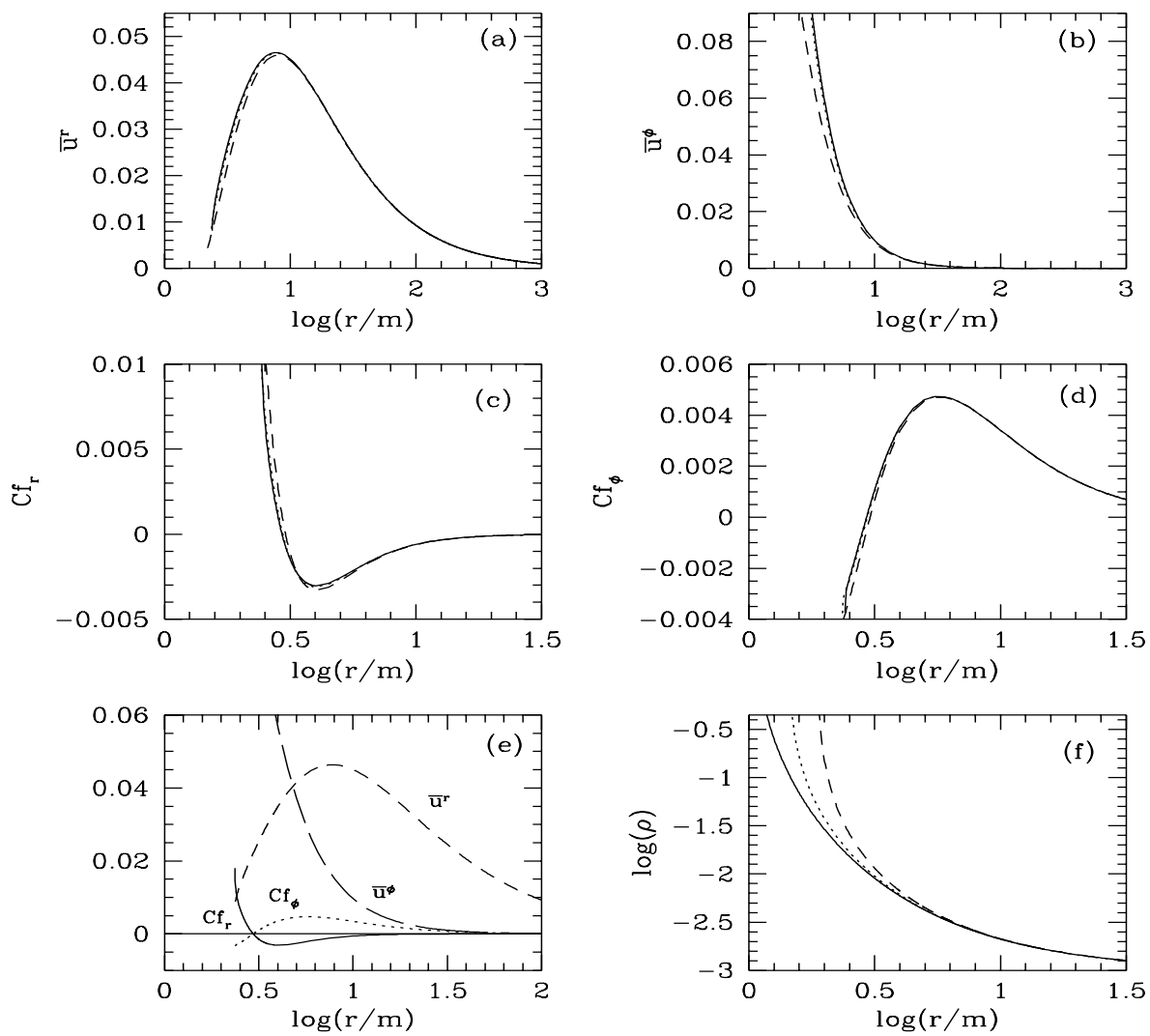

Fig. 6 : Kerr case for adiabatic equation of state with $\Gamma=4 / 3$. Plots are similar to the ones as Fig. 3.

show that though in principle there can be five roots for the equation $(C f)=0$, only two of them are outside the event horizon $r=2 \mathrm{~m}$. However the realisation of these two roots also depend upon the condition that $l$ be $\leq 3 \sqrt{3} m$. For $l=3 \sqrt{3} m$, $V \rightarrow c$, as $r \rightarrow 3 m$, which indeed is interesting, for the minimum impact parameter for a zero rest mass particle in the Schwarzschild space time is $3 \sqrt{3} m$. For this value of $l$ the centrifugal force is infinite at $r=3 m$ since the Lorentz factor goes to infinity. However, for other values of $l$ the components have two zeros, one close to $3 m$ and the other close to the horizon. The occurrence of the two zeros in the region outside the horizon does indeed depend upon the choice of the constants $l$ and $c_{1}$. The reversal for a given $l$, if occur at $r=3 \mathrm{~m}$ remains unchanged for all $c_{1}$ whereas the one nearer the horizon gets closer to the horizon with increasing values of $c_{1}$. It is also important to note that for no value of $l$ and $c_{1}$ the reversal occurs for $r>3 m$.

In the case of Kerr space time, where the equations are integrated numerically and one needs to choose the boundary conditions appropriately, it may be seen that the location of the reversal does change with the relative magnitudes of the velocity components at infinity. Table 1 gives the locations of the reversal for different values 

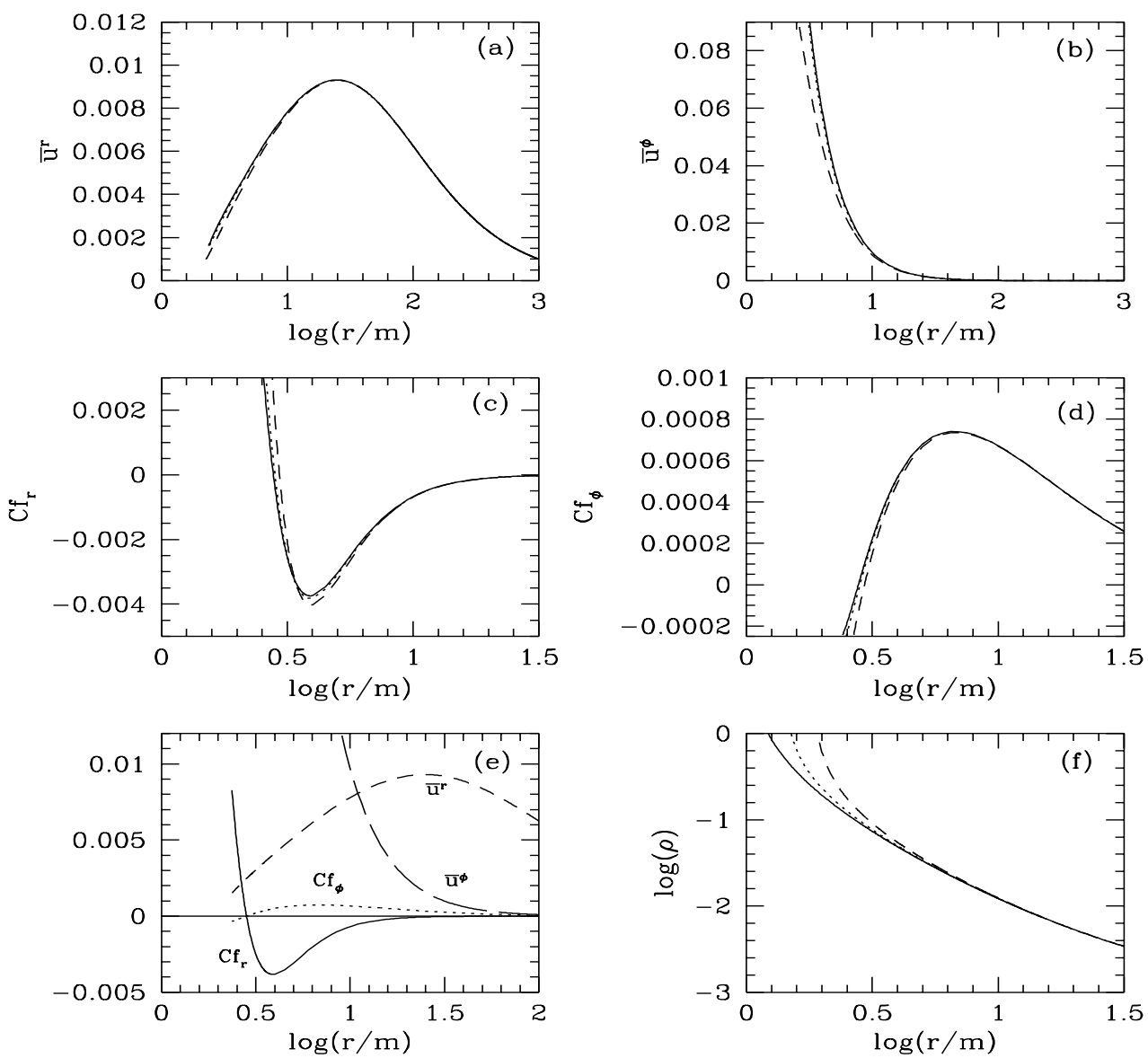

Fig. 7 : Kerr case for adiabatic equation of state with $\Gamma=5 / 3$. Plots are similar to the ones as Fig. 3.

of ' $a$ ' with corresponding boundary conditions for $\rho=$ constant case. As may be seen in the first case, $\bar{u}_{o}^{\phi}>\bar{u}_{o}^{r}$ (the subscript ' $o$ ' refers to the values at boundary from where the integration starts), and this is maintained throughout the flow with the azimuthal component dominating the centrifugal force reversal occurs at the same location as for a test particle 12 . On the other hand in the second case though $\bar{u}_{o}^{r}>\bar{u}_{o}^{\phi}$, as the flow approaches the black hole the $\bar{u}^{\phi}$ takes over $\bar{u}^{r}$ at around $r=20 \mathrm{~m}$ leading to the reversal of centrifugal force at the location for example $r=2.783 m$ for $a=0.9$ (Fig. 2e), a location slightly closer to the black hole than the reversal radius of $2.822 \mathrm{~m}$ for the test particle case. The other root seems to occur well inside the horizon and thus of no consequence. 
Table 1

$\rho=$ constant

\begin{tabular}{|c|c|c|c|c|c|}
\hline$a$ & 0 & 0.5 & 0.9 & 1 & Boundary Values \\
\hline$R$ & 3.0 & 2.944 & 2.822 & 2.783 & $\begin{array}{l}\bar{u}_{o}^{r}=10^{-8} \\
\bar{u}_{o}^{\phi}=10^{-6} \\
\rho_{o}=10^{-5}\end{array}$ \\
\hline$\overline{R_{1}}$ & 2.955 & 2.904 & 2.783 & 2.744 & $\overline{\bar{u}_{o}^{r}}=5 \times 10^{-5}$ \\
\hline$R_{2}$ & 2.117 & 1.948 & 1.596 & 1.371 & $\begin{array}{l}\bar{u}_{o}^{\phi}=10^{-6} \\
\rho_{o}=10^{-3}\end{array}$ \\
\hline
\end{tabular}

When we consider the isothermal equation of state $(P=\rho)$ in the Schwarzschild background one could solve the equations analytically yielding the velocity components in terms of two constants $v_{1}$ and $l$. As mentioned earlier $v_{1}$ can be identified as the ratio $\dot{M} / \rho$ and it is seen that the expressions for the components of centrifugal force depend upon both $v_{1}$ and $l$, with the reversal occurring at a value of $r=\frac{3 m}{2}\left(1 \pm \sqrt{1-\frac{8 v_{1}^{2}}{9 l^{2} m^{2}}}\right)$. One can straight away see from this expression that the reversal would occur at $r=3 m$ for $\frac{v_{1}}{l}<1$, which is in accordance with earlier observation as in the case where $\bar{u}^{\phi}$ would dominate over $\bar{u}^{r}$. On the other hand when $\frac{v_{1}}{l}>\frac{3 m}{2 \sqrt{2}}$ or equivalently $\frac{\dot{M}}{l \rho}>\frac{3 m}{2 \sqrt{2}}$ then the centrifugal will never be zero for any $r$ and thus no reversal would occur. This again confirms the fact that when the radial velocity dominates over the angular velocity there is no reversal of the centrifugal force. Table 2 presents the location of centrifugal reversal for the case $P=\rho$, which distinctly shows the possibility of the reversal occurring either at the same position as for a test particle or at locations different but inwards and closer to the black hole.

Table 2

$P=\rho$

\begin{tabular}{llllll}
\hline \hline$a$ & 0 & 0.5 & 0.9 & 1 & Boundary Values \\
\hline \hline & & & & & $\bar{u}_{o}^{r}=10^{-8}$ \\
$R$ & 3.0 & 2.944 & 2.822 & 2.783 & $\begin{array}{l}\bar{u}_{o}^{\phi}=10^{-6} \\
\rho_{o}=10^{-5}\end{array}$ \\
& & & & $\bar{u}_{o}^{r}=5 \times 10^{-5}$ \\
\hline \hline$R_{1}$ & 2.992 & 2.936 & 2.813 & 2.773 & $\begin{array}{l}\bar{u}_{o}^{\phi}=10^{-6} \\
R_{2}\end{array}$ \\
& No root & No root & 1.438 & 1.058 & $\rho_{o}=10^{-3}$ \\
\hline \hline
\end{tabular}

In the case of Kerr black hole one set of locations are always outside the ergosurface $r=2 m$, while the other could be inside.

Coming to the case of polytropic equation of state Table 3 presents the locations of the centrifugal reversal for different values of $a$ and of gas constant $\Gamma\left(=1+\frac{1}{n}, n\right.$ 
being the polytropic index). It is interesting to see that for $\Gamma \geq 5 / 3$ the location of centrifugal reversal coincides with those of a test particle, indicating the dominance of $\bar{u}^{\phi}$ over $\bar{u}^{r}$. On the other hand for $\Gamma, 4 / 3 \leq \Gamma<5 / 3$, the reversal occurs at locations slightly higher than those corresponding to the test particle case. As may be seen from Fig. 6e and Fig. 7e, the location of the intersection of $\bar{u}^{r}$ and $\bar{u}^{\phi}$ profiles

Table 3

$P=\rho^{\Gamma}$

\begin{tabular}{lllllll}
\hline \hline$\Gamma$ & $a$ & 0 & 0.5 & 0.9 & 1 & $n$ \\
\hline \hline $4 / 3$ & $R_{1}$ & 3.068 & 3.017 & 2.90 & 2.860 & 3 \\
& $R_{2}$ & 2.005 & 1.872 & 1.447 & 1.091 & \\
\hline \hline 1.45 & $R_{1}$ & 3.011 & 2.958 & 2.838 & 2.799 & $\sim 2.22$ \\
& $R_{2}$ & 2.003 & 1.870 & 1.444 & 1.081 & \\
\hline \hline $3 / 2$ & $R_{1}$ & 3.005 & 2.950 & 2.830 & 2.790 & 2 \\
& $R_{2}$ & 2.003 & 1.870 & 1.443 & 1.078 & \\
\hline \hline $5 / 3$ & $R_{1}$ & 3.0 & 2.945 & 2.823 & 2.783 & 1.5 \\
& $R_{2}$ & 2.002 & 1.869 & 1.442 & 1.072 & \\
\hline \hline 2 & $R_{1}$ & 3.0 & 2.945 & 2.823 & 2.783 & 1 \\
& $R_{2}$ & 2.002 & 1.868 & 1.441 & 1.067 & \\
\hline \hline
\end{tabular}

seem to have a direct bearing on the location of the centrifugal reversal. Figure 8 shows the variation of the location of centrifugal reversal as a function of the gas parameter $\Gamma$, for different values of the Kerr parameter $a$.

If we consider the Coriolis force as given by (2), it is seen immediately that while for static space time it is zero, as it should be, in Kerr space time only the radial component is non zero in Boyer-Lindquist coordinate system and is given by

$$
(C o)_{r}=-\frac{2 a m \gamma^{2} V^{\phi}}{\alpha r}\left(\frac{3 r^{2}+a^{2}}{r^{3}+a^{2} r+2 m a^{2}}\right)
$$

which matches exactly with the expression for the test particle case. This in fact is to be expected as Coriolis force does in no way depend upon the radial velocity, in the present context.

In conclusion we can say that for a fluid in curved space time, the centrifugal force reversal occurs only through the dominance of azimuthal velocity over the radial velocity. For Kerr black hole, due to the effect of inertial frame dragging, which always goes to increase the effective angular velocity, the reversal can occur even when the pressure of the fluid is zero. On the other hand when pressure is non zero, the gradient of pressure seems to bring down the effect of radial velocity and allow the fluid to have larger azimuthal velocity and thus lead to centrifugal reversal both in radial and azimuthal directions. The above discussion, however did not take into account any viscosity, which if present may affect the azimuthal velocity through the transfer of angular momentum and thus increase the effect of radial velocity. It would be necessary to extend these studies by including the 


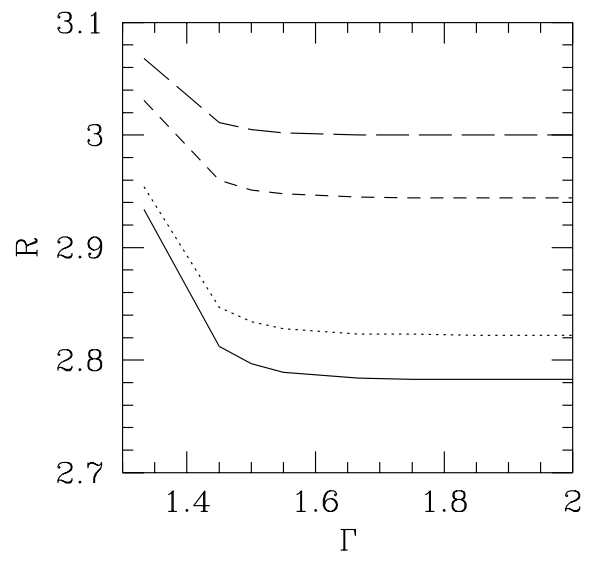

Fig. 8: Variation of the location of centrifugal reversal as a function of $\Gamma$ for Kerr parameter (a) $a=1$ (solid curve), (b) $a=0.9$ (dotted curve), (c) $a=0.5$ (dashed curve) and (d) $a=0$ (long-dashed curve).

viscous forces and analyse the effect on centrifugal reversal.

\section{References}

1. H. Bondi, MNRAS 112, 195 (1952).

2. M. Clement, ApJ 140, 1045 (1964).

3. N. R. Lebovitz, ApJ 160, 701 (1970).

4. S. Chandrasekhar \& Y. Nutku, ApJ 158, 55 (1969).

5. S. Chandrasekhar \& J. L. Friedman, ApJ 175, 379 (1972a).

6. S. Chandrasekhar \& J. L. Friedman, ApJ 176, 745 (1972b).

7. S. Chandrasekhar \& J. L. Friedman, ApJ 181, 481 (1973).

8. M. A. Abramowicz, P. Nurowski \& N. Wex, Class. Quantum Grav. 10, L183 (1993).

9. A. R. Prasanna (Submitted) (2002); gr-qc/0109020.

10. M. A. Abramowicz, B. Carter \& J. P. Lasota, Gen. Rel. Grav. 20, 1173 (1988).

11. J. W. J. York, in Gravitational Radiation, Eds. N. Deruelle \& T. Piran (North Holland, 1983), p175.

12. S. Iyer \& A. R. Prasanna, Class. Quantum Grav. 10, L13 (1993).

prepared by Latex2e October 27, 2018 